\title{
FORMACIÓN DOCENTE, PRACTICAS PEDAGOGICAS Y COMPETENCIAS DIGITALES DEL MAESTRO Y ESTUDIANTES EN LA ERA DE LA INFORMACIÓN
}

Teacher training, pedagogical practices and digital competences of the teacher and students in the age of information

\section{Judith Cristina Martínez-Royert ${ }^{1}$ María Cristina Pájaro-Martínez ${ }^{2}$}

RESUMEN : Los actuales momentos históricos por lo que atraviesa el mundo con la pandemia por covid-19, ha suscitado grandes cambios en ámbitos como educación, salud, industria, entre otros; ha provocado que la educación sea vista desde un prisma para comprender no solo la complejidad del acto educativo en sí, sino las conexiones e interconexiones de los actores que participan en él, con el acto mismo, consigo mismo, como actores protagonistas del proceso formativo y con el otro como parte fundamental; ha hecho emerger tensiones como consecuencia del rompimiento de la cotidianidad de la educación presencial y del paso hacia la educación virtual. Este artículo describe una experiencia de investigación educativa que emergió de las necesidades suscitadas por el panorama actual y cuyo objetivo general fue: implementar un proceso de innovación educativa en el curso de pregrado. se abordó desde la Investigación Acción Pedagógica; los participantes fueron 120 estudiantes del curso educación en salud (5 semestre del Programa de Enfermería) y su profesora. Ambos actores diseñaron y utilizaron de recursos educativos digitales para potencializar los procesos de enseñanza y aprendizaje a partir de ambientes motivadores e innovadores; desde la perspectiva de los actores del Proceso, la experiencia tuvo resultados calificados como excelente y bueno en relación con la valoración de

\footnotetext{
${ }^{1}$ Doctora en Ciencias de la Educación.

Profesor Investigador

Universidad Simón Bolívar. Barranquilla-Colombia

Judith.martinez@unisimonbolivar.edu.co

https://orcid.org/0000-0002-4468-8578

2 Programa de Psicología

Universidad de la Costa

https://orcid.org/0000-0002-9463-5036
} 
los recursos educativos digitales en sus características de calidad de los contenidos, interactividad, motivación, utilidad y reusabilidad y de la experiencia en general fue reconocida como valiosa y significativa por parte de los estudiantes.

Palabras clave: Formación Docente, Practicas Pedagógicas y Competencias Digitales

RESUMO : Os atuais momentos históricos pelo que atravessa o mundo com a pandemia por covid-19, tem suscitado grandes mudanças em âmbitos como educação, saúde, indústria, entre outros; levou a que a educação fosse vista de um prisma para compreender não só a complexidade do acto educativo em si, mas também as ligações e interconexões dos actores que nele participam, com o próprio acto, consigo mesmo, como protagonistas do processo formativo e com o outro como parte fundamental; fez emergir tensões como consequência do rompimento da cotidianidade da educação presencial e do passo para a educação virtual. Este artigo descreve uma experiência de pesquisa educativa que emergiu das necessidades suscitadas pelo panorama atual e cujo objetivo geral foi: implementar um processo de inovação educativa no curso de graduação. foi abordado desde a Pesquisa Ação Pedagógica; os participantes foram 120 estudantes do curso educação em saúde (5 semestre do Programa de Enfermagem) e sua professora. Ambos os atores projetaram e utilizaram de recursos educativos digitais para potencializar os processos de ensino e aprendizagem a partir de ambientes motivadores e inovadores; desde a perspectiva dos atores do Processo, a experiência teve resultados qualificados como excelentes e bons em relação à avaliação dos recursos educativos digitais nas suas características de qualidade dos conteúdos, interactividade, motivação, utilidade e reusabilidade e da experiência em geral foi reconhecida como valiosa

Palavras-chave: Formação Docente, Práticas Pedagógicas e Competências Digitais

ABSTRACT : The current historical moments that the world is going through with the pandemic by covid-19, has provoked great changes in areas such as education, health, industry, among others; has caused education to be viewed from a prism to understand not only the complexity of the educational act itself, but the connections and interconnections of the actors involved in it, with the act itself, with itself, as protagonists of the training process and with the other as a fundamental part; tensions have emerged as a result of the break with the daily life of face-to-face education and the move towards virtual education. This article describes an educational research experience that emerged from the needs raised by the current landscape 
and whose Overall objective was: to implement a process of educational innovation in the undergraduate course. was addressed from the Research Pedagogical Action; the participants were 120 students of the health education course ( 5 semester of the Nursing Program) and their teacher. Both actors designed and used digital educational resources to enhance teaching and learning processes from motivating and innovative environments; from the perspective of the actors of the Process, the experience had results qualified as excellent and good in relation to the valuation of the digital educational resources in their characteristics of content quality, interactivity, motivation, usefulness and reusability and experience in general was recognized as valuable and significant by students.

Keywords: Teacher Training, Pedagogical Practices and Digital Competencies

\section{CONSIDERACIONES INICIALES}

Los actuales momentos históricos por lo que atraviesa la humanidad con la pandemia por covid-19 ha suscitado grandes cambios en los ámbitos de la educación, salud, industria, comercio entre otros; estos cambios son de tipo Socio-estructural (revolución global y flujo migratorio), Económico-laboral (nuevas alianzas mundiales, globalización - nueva fuerza-), Tecnológico (redes universales, realidad virtual más ampliada) y cambio competencial (nuevos aprendizajes, interdisciplinariedad); todos estos cambios tienen un impacto educativo reflejado en el fácil acceso al conocimiento, provoca nuevas relaciones, exige dominio técnico, de herramientas, nuevas funciones del profesor, adaptación, trabajo en equipo, autonomía, criticidad, liderazgo, formación permanente y nuevos contenidos y métodos. En todo ello, las tecnologías de la información y comunicación (Tic) se han convertido en la mejor aliada para lograr los propósitos desde cada uno de estos ámbitos.

La pandemia ha exigido la reconfiguración de la visión de mundo que teníamos del ecosistema educativo, para el caso de los maestros o profesores, este momento histórico ha provocado que la educación sea vista desde un prisma para comprender no solo la complejidad del acto educativo en sí, sino las conexiones e interconexiones de los actores que participan en él, con el acto mismo, consigo mismo como actores protagonistas del proceso formativo y con el otro como parte fundamental; lo que ha hecho emerger tensiones como consecuencia de la fractura o rompimiento de la cotidianidad de la educación presencial y del paso hacia la educación remota/virtual. 
Este tránsito o gran salto de la presencialidad a la virtualidad, obligó a los docentes a formarse, capacitarse y /o actualizarse en ella, en la virtualidad, a reconocer las diferentes herramientas que conforman los ambientes digitales de aprendizaje y enseñanza, desde su conceptualización, pertinencia y funcionalidad; ha llevado a identificar los conceptos centrales relacionados con estos ambientes virtuales de aprendizaje (AVA) como : e-learning, aula virtual, blended learning, gamificación, herramientas digitales, ovas, podcast entre otros; para la incorporación de prácticas pedagógicas efectivas; también obligó a conocer las características y funcionalidades de las variadas herramientas y plataformas digitales utilizadas en ambientes virtuales de aprendizaje (AVA); también a resignificar las prácticas pedagógicas (PP) teniendo presente la articulación de la práctica docente (enseñanza) con la de los estudiantes (aprendizaje); además comprender su relación dialéctica lo que permite la aprehensión de la trama significados que se entrecruzan en la interacción que se da entre ellas en un ecosistema de formación, para satisfacer una necesidad y dar respuesta al encargo social de preparar para la vida a los ciudadanos planetarios (Martínez, 2019).

El nuevo contexto social exige la re-significación de las prácticas pedagógica (PP), lo que implica importantes transformaciones de ellas, a partir del conocimiento y habilidades tecnológicas que debe tener el maestro (Hernández, 2017); esta realidad será posible desde una formación docente permanente, responsable y pertinente a los nuevos contextos de enseñanza, aprendizaje y evaluación; donde las competencias digitales tienen un rol transversal en los procesos formativos, que requiere ser desarrolladas por estudiantes y maestros (profesores).

Este artículo pretende presentar, de manera contextualizada, una experiencia investigativa de aula, desarrollado desde el programa de pregrado de enfermería de una universidad privada del caribe colombiano; se muestran resultados significativos de una práctica pedagógica (PP) mediada por la tecnología de información y comunicación (tics), que permitió el desarrollo exitoso de competencias digitales en maestros y estudiantes del programa de Enfermería.

\section{FUNDAMENTACIÓN TEÓRICA}

\section{ENSEÑANZA Y APRENDIZAJE EN ENTORNOS VIRTUALES: RETOS EN LA ERA INFORMÁTICA}


Ante una nueva ecología del ambiente educativo provocado por el momento histórico actual (Pandemia por covid-19), el profesor de hoy está obligado a re-significar sus PP en lo relacionado con la inclusión de nuevas didácticas, en la que las tics (tecnología de la información y comunicación), son un factor inherente al tránsito entre conocimiento y sociedad y ocupan un lugar predominante para desarrollar los procesos de enseñanza, aprendizaje y evaluación del aprendizaje. La re-significación implica repensar, transformar e innovar el acto educativo, reinventarse estrategias de enseñanza que tengan como alidada las tecnologías para propiciar escenarios de aprendizaje motivadores, innovadores y creativos que potencien el aprendizaje efectivo y significativo y a su vez incentiven el uso de ellas en los discentes a partir del reconocimiento de los aportes que la tecnología ofrece al campo sanitario.

El uso de las Tic en el ámbito educativo ha experimentado variedad de cambios, agudizados por las actuales circunstancias pandémicas, pero también por las complejidades de las necesidades educativas que plantea la actual sociedad del conocimiento que va de la mano de los avances tecnológicos, lo que pone en evidencia las tendencias actuales por las que cursa la educación donde las tecnología se posicionan en un lugar protagónico y estratégicos para lograr la universalidad, la masificación de la educación y mayor accesibilidad a ella; al trascender tiempo y espacio, y proporcionando los estudios a personas que por su condición o característica particular, le impidan una formación presencial o semipresencial. Cabe señalar que, en la virtualidad, se destaca el uso educativo de dispositivos móviles, lo que "aumenta las ventajas propias del aprendizaje flexible al romper aún más las barreras espaciotemporales" (García, 2017, p. 20). Las tecnologías han impactado de una manera significativa el quehacer pedagógico y a la vez la experiencia de su uso en los procesos educativos ha llevado a la reflexión acerca de las pedagogías emergentes del entorno virtual.

\section{COMPETENCIAS DIGITALES EN LA FORMACIÓN ACTUAL DEL PROFESIONAL EN FORMACIÓN}

La competencia digital (CD) es aquella en la que el uso creativo, crítico y seguro de las tecnologías de la información y la comunicación es imprescindible para alcanzar los objetivos relacionados con el trabajo, el aprendizaje, el uso del tiempo libre, la inclusión y participación en la sociedad (Villarreal-Villa, García-Guliany, Hernández-Palma y Steffens-Sanabria, 2019). 
Gracias a las tecnologías se ha logrado el empoderamiento de la información lo que ha conducido a cambios importante en todos los contextos de la sociedad y ha generado de manera acelerada transformaciones significativas en ellos. La integración y articulación de las tecnologías a los procesos educativos ha legitimado el desarrollo de medios y herramientas digitales y los ambientes virtuales de aprendizaje (AVA) en donde los Recursos Educativos Digitales (RED) y los Objetos Virtuales de Aprendizaje (OVAS) son considerados, por los expertos, como herramientas relevantes que miden los procesos de enseñanza, aprendizaje y evaluación de los aprendizajes Galindo, Ruiz, y Ruiz (2017).

Ante los desafíos que ha traído la pandemia por covid-19, por mantener y mejora la calidad educativa en estos nuevos entornos de aprendizaje virtuales, las organizaciones educativas se dieron a la tarea de adquirir plataformas, software y capacitar al talento humano (profesores) en todo lo relacionado con la virtualidad para aunar esfuerzos y que no se viera afectada la calidad de los procesos formativos, todo ello se dio sobre la marcha, nadie estaba preparado para este momento histórico que a todos nos tomó por sorpresa. Lo que exigió a las organizaciones educativas actualizarse en herramientas virtuales necesarias para transitar hacia la virtualidad como parte de una "nueva cotidianidad" y llevó a utilizar a profundidad metodologías activas, considerada como un proceso dinámico donde el estudiante es el protagonista de su proceso educativo y el docente o profesor asume el rol de tutor o guía; La metodología activa es una propuesta que se opone a los métodos pasivos de la educación, en los cuales el docente solo es transmisor de la información y el estudiante un receptor (Silva y Maturana, 2017), (Robledo et al, 2015), (da Costa, 2019)

En este sentido y teniendo en cuenta el contexto actual, que ha llevado a incursionar a todas las organizaciones educativas en la educación virtual y remota, este artículo describe una investigación educativa que emergió de las necesidades suscitadas por el estado actual y cuyo objetivo general fue: implementar un proceso de innovación educativa en el curso de Educación en Salud del programa de Enfermería del Universidad Simón Bolivar-Barranquilla -Colombia.

\section{METODO}

La presente investigación se desarrolló durante el año 2020 en el curso de Educación en Salud, este curso se enfoca en el rol educativo del profesional de Enfermería en concordancia con el perfil social del Programa y el Proyecto Educativo Institucional (PEI) de la Universidad 
Simón Bolívar (USB), incluye aspectos relacionados a los procesos didácticos y evaluativos concernientes a garantizar una dimensión teórica del proceso de salud- Enfermedad y aportes conceptuales de estrategias transversales de intervención en salud; además el curso describe y aborda diversas formas de construir colectivamente estrategias educativas que propendan por el bienestar individual y comunitario, ya sea para prevenir factores de riesgos prevalentes o promover estilos de vida saludables.

\section{DISEÑO}

Es una investigación de aula, del tipo Investigación acción pedagógica, tuvo en cuenta el replanteamiento de los modelos de formación que busca posicionar un maestro investigador que desarrolla investigación e innova en su práctica pedagógica; es decir que el profesor transforma su práctica desde la investigación, llevando el currículo al laboratorio de las aulas (Lawrence Stenhouse, 1984); (Lewin Kurt, 1948); la puesta de esta experiencia investigativa fue desarrollar el curso desde unas prácticas pedagógicas apoyada de las tecnologías de la información y la comunicación (TIC), donde ambos actores Profesor - estudiante tuvieran un rol protagónico en el Proceso.

\section{POBLACIÓN}

Los participantes fueron los estudiantes del programa de Enfermería de V semestres matriculados en el curso (120 estudiantes) y la profesora a cargo del desarrollo del Curso.

\section{DESCRIPCIÓN DE LA EXPERIENCIA}

Teniendo en cuenta el propósito principal de la presente investigación, implementar un proceso de innovación educativa en el Curso, además de potenciar las competencias propias del curso, se buscó desarrollar competencias digitales en el enfermero en formación. En su desarrollo, se utilizó de manera intensiva las TIC, se diseñaron Recursos Educativos Digitales (RED) permitiendo en todo momento comunicación sincrónica o asincrónica, entre el profesor y los estudiantes, haciendo uso, para ello, de la plataforma de la universidad Moodle versión 
3.8.2, Plataforma Teams, Herramienta H5P (moodle) y del software en línea Genially, Menti.com.

\section{MOMENTOS DE LA INVESTIGACIÓN}

Esta experiencia se desarrolló a través de las siguientes fases o momentos sintetizados en la figura 1.

Figura 1. Produción propia.

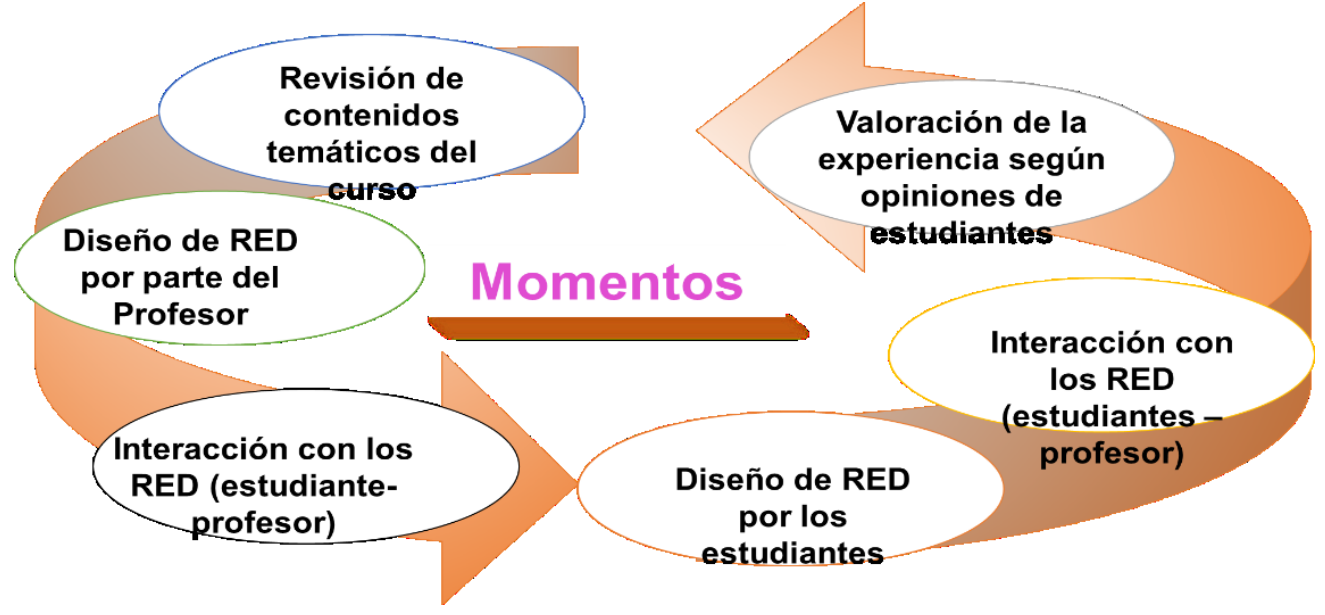

Fuente: Elaboración propia

\section{RESULTADOS}

A continuación, se relacionan algunos Recursos Educativos Digitales (RED) diseñados por la profesora del Curso.

\section{RECURSOS EDUCATIVOS DIGITALES CREADO POR LA DOCENTE}

Figura 2. Produción propia. 


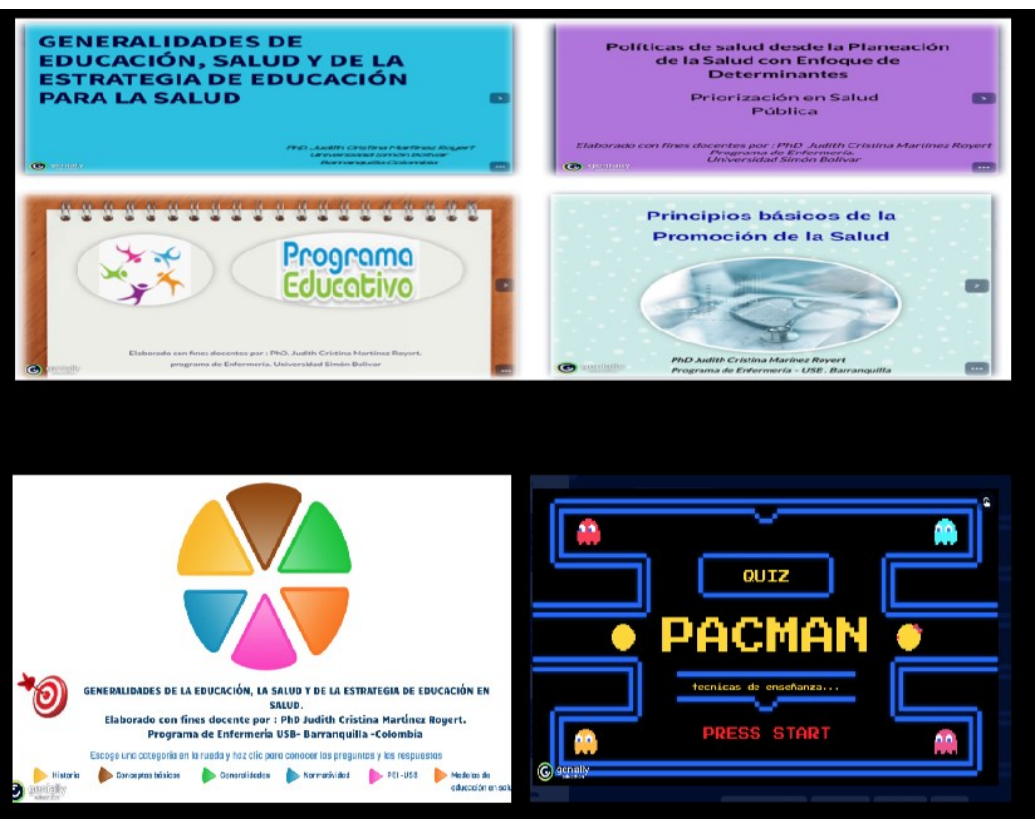

Fuente: Elaboración propia.

Desde el inicio del curso se planificó y aplicó el uso de los RED incluyendo la inducción o presentación del curso, fueron diseñados con el software Genially, exelearning y otras herramientas como H5P usadas con el fin de motivar y potencializar el aprendizaje de los estudiantes publicados en el aula extendida (aula virtual- plataforma Moodle), (ver figura 2)

Figura 2. Red elaborados por la profesora con herramientas H5P, publicados en el aula extendida (aula virtual).

Figura 3. RED.

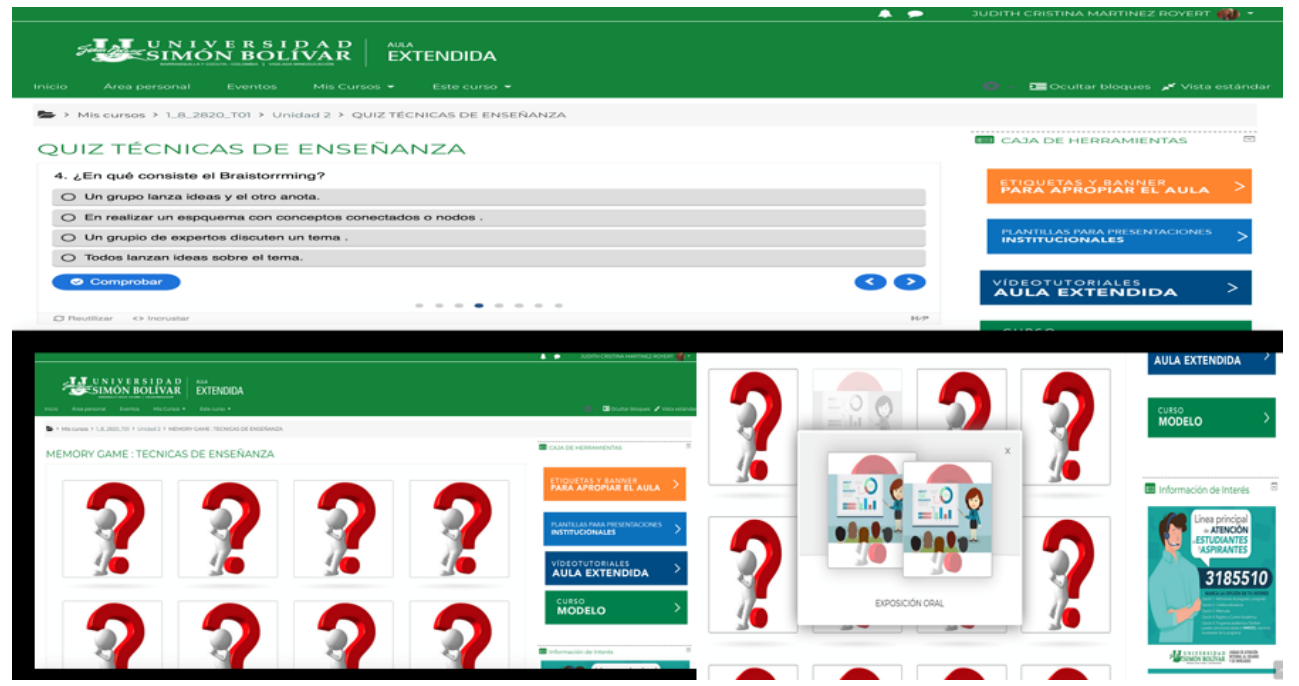


Fuente: Universidad Simón Bolivar.

Los estudiantes fueron motivados para construir sus propios RED como parte de algunas actividades académicas, entre ellas se resalta los contenidos del programa educativo en donde ellos como trabajo final diseñan un programa educativo dirigido a una población específica, en este caso fueron escolares de 4 y 5 de primaria, donde como apoyo para realizar las sesiones educativas diseñaron algunos RED final del curso (figura 4) y (figura 5).

Figura 4. RED diseñada por estudiantes para el Programa Educativo dirigido a escolares.

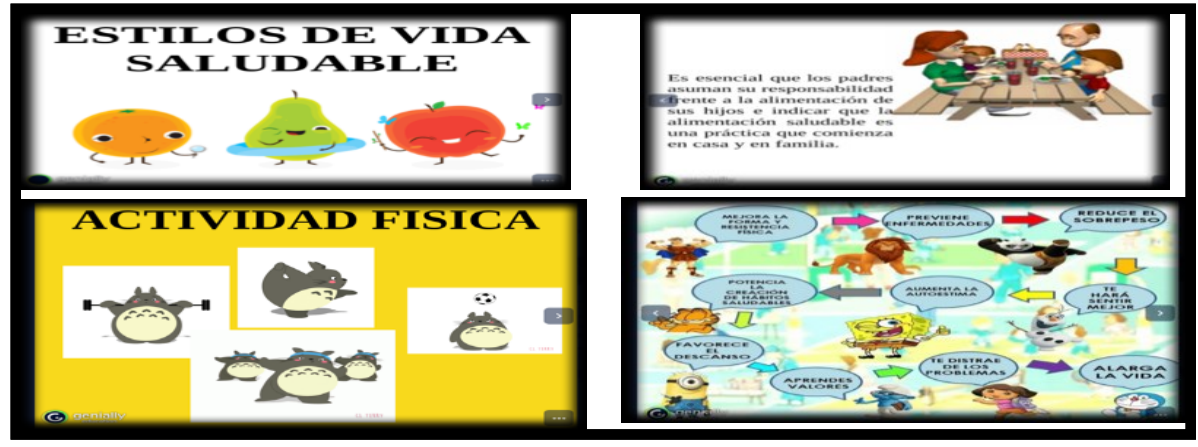

Fuente: elaborado por estudiantes con el software libre Genially.

Figura 5. Infografías interactivas diseñadas por estudiantes sobre promoción de la salud y educación para la salud 


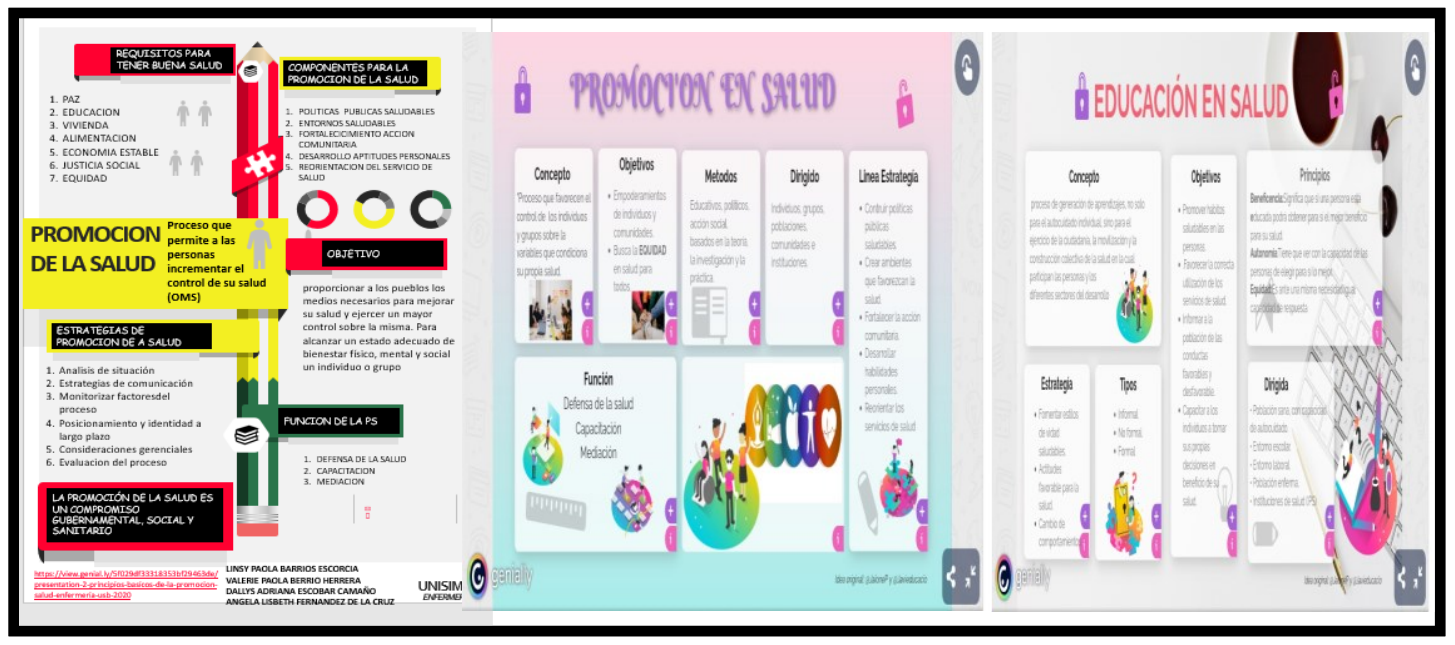

Fuente: elaboración por estudiantes.

Los recursos educativo digitales fueron colgados en el aula extendida (Plataforma Moodle y Microsoft Teams, con el fin de que los estudiantes interactuaran durante el curso y reforzaran los contenidos temáticos abordados, incluyendo los creados por los estudiantes como parte de su proceso y diseño del programa educativo dirigido a escolares con dos temas: alimentación saludable, actividad física y promoción de la salud

Como parte de la autorregulación del proceso y luego de avanzar en el desarrollo del curso, se aplicó un instrumento (lista de chequeo) a los estudiantes para conocer su opinión acerca de los RED en cuanto a las siguientes dimensiones: Calidad de los contenidos, interactividad, motivación diseño, reusabilidad, la cual fue muy favorable, lo que evidencia una alta aceptación (figura 6).

Figura 6. Opiniones de los estudiantes en relación a la experiencia con la utilización de los RED como práctica innovadora en el desarrollo del curso 


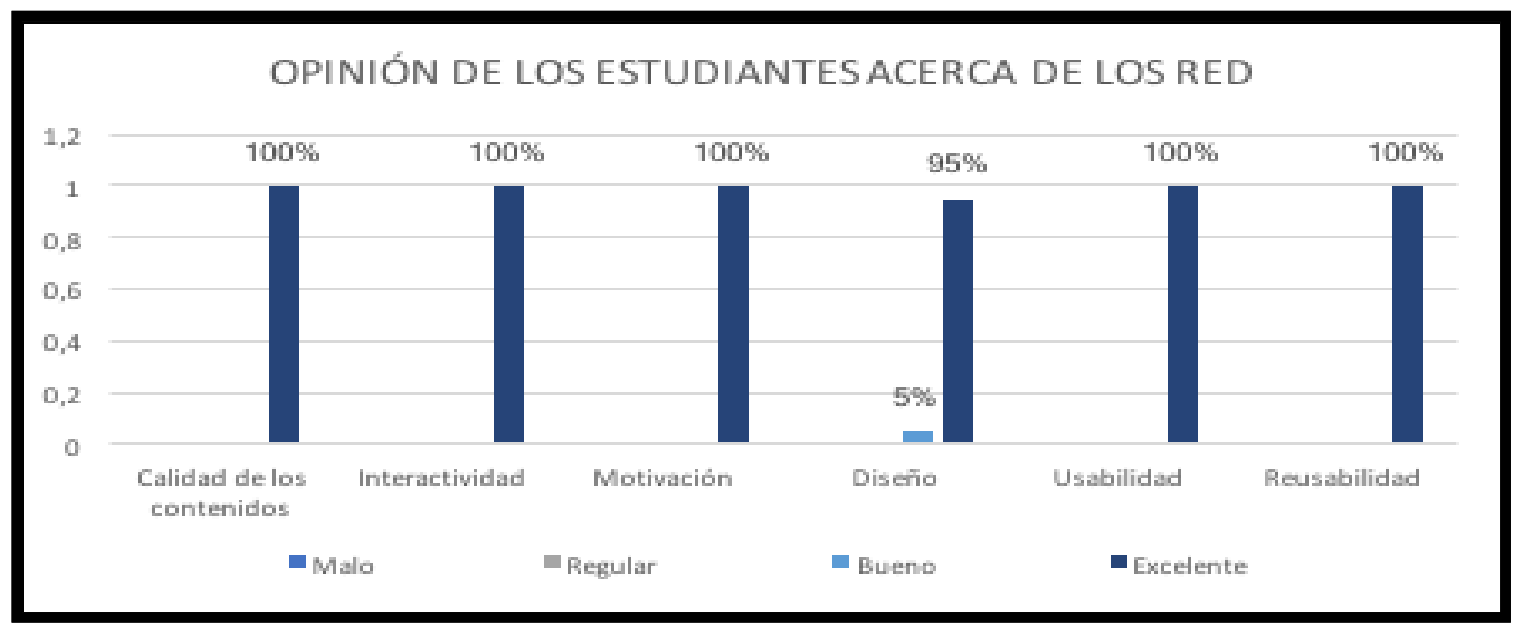

Fuente: Instrumento aplicado.

La dimensión de Calidad de los contenidos hace relación a que los RED brindaron una información clara con una buena presentación del contenido/actividad. La interactividad, responde a la dinámica de la presentación de los contenidos adaptables a diferentes tipos de usuario (estudiantes y docentes). Con respecto a la Motivación, se les indagó acerca si el contenido de los RED les atraía para aprender y adquirir nuevos conocimientos de manera atractiva y despertaba su interés. El diseño, hace referencia a la forma como se presentó cada contenido en el recurso digital. Usabilidad, en esta dimensión se les preguntó por la facilidad con la que interactuaron con el RED; por último, se valoró la reusabilidad, entendida esta como la capacidad y posibilidad de usar el RED varias veces, el número de ocasiones que el estudiante lo crea necesario para reforzar su aprendizaje. Se destaca que todos los aspectos fueron valorados como excelente del 100\%, excepto el diseño donde 95\% manifestó excelente y el 5\% lo valoró como bueno.

Los estudiantes reconocieron el valioso aporte de las tecnología y el protagonismo que han tenido las tics como consecuencia del COVID-19 y sus continuos avances, resaltaron, para el desarrollo del curso educación en salud, tributos como la accesibilidad a la información, la innovación herramienta, comunicación permanente que contribuyen a un aprendizaje autónomo, autodirigido

\section{DISCUSIÓN Y CONCLUSIONES}


Los cambios actuales en el campo de la educación y la salud que se han venido suscitando debido a la evolución del conocimiento, la sociedad misma, los avances tecnológico, y que hoy, con el momento histórico que se vive, en el contexto de la Pandemia por covid-19, se han generado profundas transformaciones en la manera como se asume la práctica en estos dos campos; en donde las tecnologías se han convertido en una herramienta primordial para continuar desarrollando acciones tanto en la educación como en la salud.

En este sentido es pertinente destacar las contribuciones de la tecnología en la práctica educativa, donde hoy se enseña utilizando diversas plataformas con apoyo de múltiples herramientas para generar espacios de aprendizaje: Desde esta experiencia investigativa en el campo de la educación se resalta, el valor significativo, para el caso de esta investigación de aula, de los RED como un apoyo para propiciar espacios motivadores, innovadores, y efectivos en el aprendizaje, desde la enseñanza y la evaluación teniendo en cuenta la diversidad de estilos de aprendizaje presentes en los estudiantes para propiciar empoderamiento del conocimiento compartido (Martínez- Royert, 2019); (Martínez-Royert y Pájaro-Martínez, 2020). La incorporación de las tecnologías en la enseñanza tuvo una aceptación y favorabilidad en cuanto al aprendizaje mismo, la motivación por parte de los estudiantes, estos resultados coinciden con los realizados por Barroso-Osuna, Julio, Cabero-Almenara, Julio, \& Gutiérrez-Castillo, JuanJesús (2018) y Ramírez y Espinoza (2017) donde la aplicación de tecnología en los cursos de programas universitarios demostró un nivel de satisfacción elevado por pate de los estudiantes.

La didáctica con el apoyo de la tecnología, se han configurado como "didáctica alternativa", donde los contenidos y métodos de enseñanza son permeados por la virtualidad transformando la manera como, convencionalmente, se ha venido desarrollando el proceso de formación en el aula física promoviendo y desarrollando competencias de mayor complejidad en el estudiante como el pensamiento crítico y creativo; lo que coincide con la postura de Aguilar , 2015, p 357 cuando afirma que durante el proceso de enseñanza se emplea la didáctica tecnológica (didáctica apoyadas de la tics) la inmediatez de la información posibilita " un alto protagonismo a los participantes como creadores y recreadores de su propia cultura gracias a esa interconexión ágil, flexible, interactiva, asíncrona y de tiempos fluidos que supone lo virtual".

Está demostrado que la tecnología es un elemento transcendental para el desarrollo de habilidades en los estudiantes del siglo XXI, y cumple un papel clave y fundamental en la construcción del conocimiento; el uso de ella permite acceder al conocimiento global y a su vez transformar entornos con la aprehensión de ella e integración del conocimiento glocal. 
Las herramientas digitales son recursos poderosos a favor de la educación, bien fundamentadas dentro de un plan educativo pueden enriquecer las experiencias de aprendizaje del estudiante y facilitar la labor del educador. Las tecnologías se han convertido en un reto actual para el proceso de formación de un nuevo profesional de enfermería lo que representa la necesidad de repensar tanto la formación del futuro profesional y tener en cuenta las competencias digitales que debe desarrollar para estar acorde con los actuales y futuros contextos, porque la tecnología llegó para quedarse y es que el uso de las TIC con el transcurrir de los años y más aún, en los actuales momentos, se convirtió en una herramienta imprescindible en la salud, en la educación y otros campos disciplinares y profesionales (Rivero y Choperena, 2019).

\section{REFERENCIAS}

Aguilar, D. (2015). Ser docente virtual: tiempo y presencia (Tesis doctoral). Universidad de Málaga, España.

Barroso-Osuna, Julio, Cabero-Almenara, Julio, \& Gutiérrez-Castillo, Juan-Jesús. (2018). La producción de objetos de aprendizaje en realidad aumentada por estudiantes universitarios. Grado de aceptación de esta tecnología y motivación para su uso. Revista mexicana de investigación educativa, 23(79), 1261-1283. Recuperado en 21 de agosto de 2020, de

http://www.scielo.org.mx/scielo.php?script=sci_arttext\&pid=\$140566662018000401261\&lng $=$ es\&tlng=es.

Colpas, P., Tapias, B., Comas, A., Melo, M., \& Martinez-Royert, J. M. (2020). Aula Touch Game: Digital Tablets and Their Incidence in the Development of Citizen Competences of Middle Education Students in the District of Barranquilla-Colombia. Advances in Swarm Intelligence: 11th International Conference, ICSI 2020, Belgrade, Serbia, July 14-20, 2020, Proceedings, 12145, 537-546. https://doi.org/10.1007/978-3-030-53956-6_49

Da Costa, D. P. (2019). Gincanas Como Espaço de Formação Docente: das (inter) faces possíveis em um curso de Pedagogia. Cadernos Cajuína, 4(1), 84-104.

García, L. (2017). Educación a distancia y virtual: calidad, disrupción, aprendizajes adaptativo y móvil. Revista Iberoamericana de Educación a Distancia, 20(2), 09-25. doi: http://dx.doi.org/10.5944/ried.20.2.18737

Galindo, F., Ruiz, S., \& Ruiz, F. (2017). Competencias digitales ante la irrupción de la Cuarta Revolución Industrial. Estudos em Comunicação, 25(1), 1-11. Recuperado de: http://ojs.labcom-ifp.ubi.pt/index.php/ec/article/view/277 
Lewin, K., 1948, Resolving Social Conflicts; Selected Papers on Group Dynamics, Gertrude W. Lewin ed., Nueva York, Harper and Row.

Hernández, R.M. (2017). Impacto de las TIC en la educación: Retos y Perspectivas. Propósitos y Representaciones, 5(1), $325 \quad$ - 347. http://dx.doi.org/10.20511/pyr2017.v5n1.149

Martínez-Royert JC. (2019). La evaluación: una práctica docente compleja. En Prácticas pedagógicas complejas. Un encuentro con la realidad. González Velasco (coord). Beltrán impresiones \& Estrategias. ISBN 978-99974-0631-6 p 57-64.

\& Pájaro-Martínez, M. C. (2020). Relación entre los estilos y estrategias de aprendizaje en estudiantes de facultad de ingeniería de una Universidad en Colombia. Revista Boletín Redipe, 9(9), 147-163.DOI https://doi.org/10.36260/rbr.v9i9.1073

Organización Mundial de la Salud. OMS. Organización Panamericana de la Salud. OPS. Agenda de Salud Sostenible para las Américas 2018-2030: Un llamado a la acción por la salud y bienestar de las Américas. 9.a CONFERENCIA SANITARIA PANAMERICANA. 25 de septiembre del 2017. CE154/1CSP29/6, Rev. 3*. Online http: / / www.paho.org/hq/index.php?option $=$ com_content\&view $=$ article\&id $=13246 \% 3 \mathrm{Aheal}$ th-agenda americas\&catid $=9277 \% 3$ Acontents\&Itemid $=42349 \&$ lang $=\mathrm{es}$

Ramírez, M. M. O., \& Espinosa, Z. S. M. (2017). Evaluación de estrategias para promover el aprendizaje usando TIC: el caso de un curso de Temas de Estadística Multivariante. Revista Tecnología, Ciencia y Educación, (6).

Rivero, R. M. R., \& Choperena, C. I. P. (2019). Tecnología, globalización e investigación en enfermería: aproximaciones para un nuevo modelo de formación profesional. Cultura del Cuidado Enfermeria, 16(1), 89-98.

Robledo, Patricia; Fidalgo, Raquel; Arias, Olga; Álvarez, Ma Lourdes (2015). Percepción de los estudiantes sobre el desarrollo de competencias a través de diferentes metodologías activas. Revista de Investigación Educativa, 33(2), 369-383. DOI: http://dx.doi.org/10.6018/rie.33.2.201381

Rossi Rivero, R., \& Padilla Choperena, C. (2019). Tecnología, globalización e investigación en enfermería: aproximaciones para un nuevo modelo de formación profesional. Cultura Del Cuidado, 16(1), 89-98. Recuperado a partir de https://revistas.unilibre.edu.co/index.php/cultura/article/view/5848

Silva Quiroz, Juan, \& Maturana Castillo, Daniela. (2017). Una propuesta de modelo para introducir metodologías activas en educación superior. Innovación educativa (México, DF), 17(73), 117-131. Recuperado en 15 de enero de 2021, de http:/ /www.scielo.org.mx/scielo.php?script=sci_arttext\&pid=S166526732017000100117\&lng $=$ es\&tlng $=$ es. 
Stenhouse, L., 1984, Investigación y desarrollo del curriculum, 4.a ed., Madrid, Morata.

Villarreal-Villa, S., García-Guliany, J., Hernández-Palma, H., y Steffens-Sanabria, E., (2019). Competencias docentes y transformaciones en la educación en la era digital. Form. Univ, 12(6), 3-14 https://doi.org/10.4067/S0718-50062019000600003 